\title{
Applications de la dissolution totale des éléments colloïdaux du lait à la détermination de son activité protéinasique
}

\author{
par \\ G. HUMBERT*, R. DESNOUVEAUX* et G. LINDEN*
}

\section{Rés u m é}

Les activités protéinasiques des laits crus ont été mesurées sur 10 substrats de synthèse de type 4-nitroanilide et en se servant d'un mélange dissolvant (Butylamine, Cyclohexanone et Triton X-100: 2/1/1) des constituants colloïdaux du lait.

Les cinétiques des activités présentent de fortes variations d'un substrat à l'autre et aucune corrélation ne se dégage qui permette d'expliquer l'origine de l'activité protéinasique.

Mots clés

Dissolution - Lait - Protéinases - Flores bactériennes.

Titre abrégé

Activités protéinasiques après dissolution du lait.

\section{S u m m a ry}

APPLICATIONS OF TOTAL DISSOLUTION OF COLLOIDAL COMPONENTS OF MILK TO PROTEINASIC ACTIVITY DETERMINATION

The proteinasic activities in raw milk were measured on ten 4-nitroanilid substrates by mean of the mixture (butylamine, cyclohexanone and Triton $X-100: 2 / 1 / 1)$ to dissolve the milk colloïdal components.

* Service de Biochimie Appliquée, Université de Nancy-1, 54506 Vandœuvre-lèsNancy Cedex. 
The kinetics of activities showed great differences between the substrates. Correlation could not be established to determine the origin of the proteinasic activity.

Key-words

Dissolution - Milk - Proteinases - Bacterial population.

Run title

Proteinasic activities in transparent milk.

\section{INTRODUCTION}

Après bien des controverses, il est désormais bien établi que le lait renferme un système protéinasique natif, auquel s'ajoutent éventuellement des protéinases d'origine bactérienne. Leur activité peut avoir des incidences en technologie laitière : gélification des laits et crèmes U.H.T., modification des caractéristiques organoleptiques de produits laitiers (Humbert et Alais, 1979 ; Fox, 1981 ; Visser, 1981).

Dans l'activité protéinasique endogène du lait, on distingue différents types d'activité : thrombine, plasmine (Reimerdes, Halpaap et Klostermeyer, 1981), aminopeptidases (Reimerdes, Petersen et Kielwein, 1979), cathepsine (Kaminogawa et Yamauchi, 1972). Dans tous les travaux, l'activité dominante se manifeste à $\mathrm{pH}$ alcalin.

Les méthodes classiques de détermination de l'activité protéinasique (mesure de la libération de l'azote non protéique après incubation aseptique, analyse densitométrique d'électrophorégrammes des caséines) sont longues; de plus, les autres méthodes faisant appel à une mesure au spectromètre d'absorption moléculaire sont difficilement applicables de façon directe à un milieu opaque comme le lait.

Dans un travail récent (Linden et al., 1982), nous avons décrit un protocole de mesure directe de l'activité protéinasique du lait à l'aide de substrats de synthèse en employant un mélange dissolvant de ses constituants colloïdaux. Nous avons voulu éprouver dans cette étude ce nouveau protocole en testant sur 9 laits crus une série de substrats dans le but de mettre en évidence des corrélations avec la méthode conventionnelle de détermination à l'azote non protéique. Le deuxième objectif est de pouvoir apprécier indirectement la qualité hygiénique d'un lait.

\section{MATERIEL ET METHODES}

Le protocole de mesure de l'activité protéinasique a été décrit précédemment (Linden et al., 1982). Les mesures sont effectuées à $37^{\circ} \mathrm{C}, \mathrm{pH} 8$ avec les substrats en concentration finale de $5.10^{-3} \mathrm{~mol} / 1$ 
(sauf $3.10^{-3} \mathrm{~mol} / 1$ pour L-Phénylalanine 4-nitroanilide). Le coefficient de variation des résultats est égal à $5 \%$.

La mesure classique de l'activité protéinasique s'inspire de la méthode adaptée par Noomen (1975). On détermine l'augmentation de l'azote non protéique (NPN) dans le lait après 5 jours d'incubation à $37^{\circ} \mathrm{C}$ en présence de merthiolate $(0,12 \%)$ comme agent anti-bactérien. Les dosages d'azote sont faits par microanalyse Kjeldahl. L'azote non protéique est déterminé sur le filtrat trichloracétique à $12 \%$ de concentration finale avant et après incubation et exprimé en pourcent de l'azote total.

Les déterminations des flores mésophiles et psychrotrophes ont été faites selon Serres, Amariglio et Pétransxiène (1974).

Nous avons testé 7 laits crus individuels prélevés aseptiquement et 2 laits de mélange. L'un des laits de mélange a subi une contamination bactérienne naturelle en séjournant à l'air libre dans l'étable pendant quelques heures.

Tous les réactifs utilisés sont de la catégorie " pour analyses » ou d'une pureté équivalente. Les réactifs suivants proviennent de Prolabo, Paris : triéthanolamine (TEA), triton X-100, cyclohexanone, azoture de sodium et diméthylformamide. La n-butylamine a été fournie par Merck, Darmstadt, R.F.A. Les substrats fournis par Serva, Heidelberg, R.F.A. sont : L-Alanine-4-nitroanilide (L-Ala-4 na), N-acétyl-L-Alanine4-nitroanilide (N-Ac-Ala-4 na), N- $\alpha$-Benzoyl-DL-Arginine-4-nitroanilide (DL-Bapa), Glycine-4-nitroanilide (Gly-4 na), L-Leucine-4-nitroanilide (L-Leu-4 na), L-Lysine-4-nitroanilide (L-Lys-4 na), L-Phénylalanine-4nitroanilide (L-Phe-4 na), N-glutaryl-L-Phénylalanine-4-nitroanilide (GluPhe-4 na), N-acétyl-L-Tyrosine-4-nitroanilide (N-Ac-Tyr-4 na).

\section{RESULTATS ET DISCUSSION}

\section{Lait cru de mélange}

Cette étude a été faite avec 10 substrats différents, tous de la famille des 4-nitroanilides. Dans nos conditions opératoires (température, concentration en substrats), les cinétiques d'hydrolyse sont linéaires jusqu'à 24 h (fig. 1). Au-delà, les courbes s'incurvent ensuite différemment selon les substrats.

Pour les substrats L-Leu-4 na et L-Ala-4 na, la phase linéaire peut être inférieure à $24 \mathrm{~h}$ dans le cas d'un lait dont l'activité enzymatique est particulièrement élevée. Ces résultats nous ont conduits à effectuer les mesures à $24 \mathrm{~h}$ maximum et avec une mesure ponctuelle à $6 \mathrm{~h}$.

Deux substrats: DL-Blpa et N-Ac-Tyr-4 na ne sont pas du tout hydrolysés. Il est surprenant de constater que les substrats L-Leu-4 na et L-Ala-4 na soient plus hydrolysés que des substrats comme le DL- 


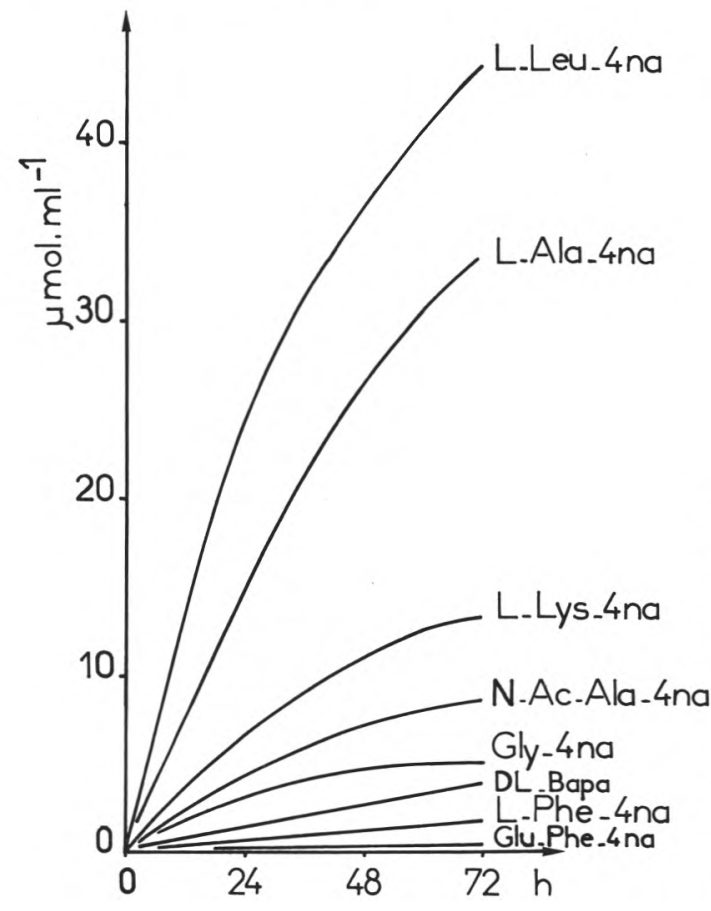

fig. 1

Cinétique d'hydrolyse de substrats de type 4-nitroanilide dans un lait cru de mélange (concentration en substrat $5.10^{-3} \mathrm{~mol} / \mathrm{l}$ sauf pour L-Phe-4 na : $3.10^{-3} \mathrm{~mol} / \mathrm{l}$ ).

Kinetic of hydrolysis of 4nitroanilide substrates in raw milk (substrate concentration: $5.10-3 \mathrm{~mol} / \mathrm{l}$ except for L-Phe-4 na: $\left.3.10^{-3} \mathrm{~mol} / \mathrm{l}\right)$.

\section{TABLEAU 1 - TABLE 1}

Contenu en germes microbiens des laits crus individuels (par ml)

Bacterial population in the individual raw milks (c.f. $u / m l$ )

\begin{tabular}{c|c|c}
\hline Laits & Flore mésophile & Flore Psychrotrophe \\
1 & 2650 & 610 \\
2 & 1830 & 360 \\
3 & 2150 & 310 \\
4 & 6400 & 840 \\
5 & 6000 & 1040 \\
6 & 2860 & 510 \\
7 & 9700 & 2390 \\
\hline
\end{tabular}


Blpa et DL-Bapa qui sont spécifiques des activités du type thrombine et plasmine. Nos résultats sont divergents de ceux rapportés par Reimerdes, Petersen et Kielwein (1979). Cette étude devra être complétée par l'emploi de substrats plus spécifiques d'endopeptidases.

\section{Laits crus individuels}

Cette étude a concerné 7 laits crus individuels dont le contenu microbien est rapporté dans le tableau 1. Les laits sont peu contaminés ; la teneur en germes est toujours inférieure à 10000 germes $/ \mathrm{ml}$.

Cette étude a été faite avec 6 substrats de synthèse choisis soit pour leur spécificité, soit pour leur intensité de réponse. Nous rapportons sur la figure 2 l'activité protéinasique des laits mesurés en suivant l'hydrolyse de ces 6 substrats et l'augmentation du NPN. Nous retrouvons les plus fortes valeurs de protéolyse pour les 3 substrats L-Leu-4 na, L-Ala-4 na et L-Lys-4 na. Il ne semble pas y avoir de corrélations entre les 2 méthodes de mesure. De plus, le profil des activités

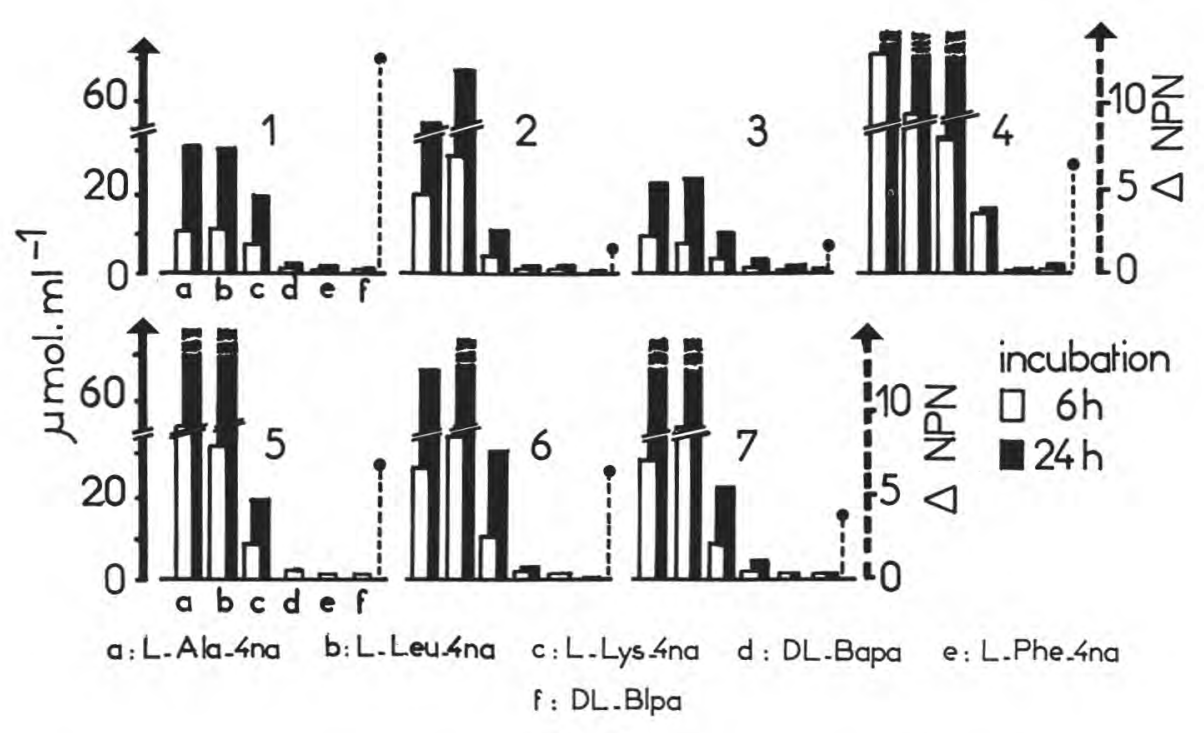

fig. 2

Comparaison de deux méthodes de détermination de l'activité protéinasique : dosage du NPN et hydrolyse des substrats (concentration en substrat : $5.10^{-3}$ mol/l sauf pour L-Phe-4 na : $\left.3.10^{-3} \mathrm{~mol} / \mathrm{l}\right)$.

Comparison of two methods of proteinasic activity determination: NPN measurement and substrates hydrolysis (substrate concentration: $5.10-3 \mathrm{~mol} / \mathrm{l}$ except for L-Phe-4 na: $\left.3.10^{-3} \mathrm{~mol} / \mathrm{l}\right)$. 
enzymatiques ne rend pas compte des écarts de la flore bactérienne entre les échantillons.

Outre le fait que l'on peut lui reprocher d'être longue, la mesure de la libération de l'azote non protéique n'est pas la méthode la plus adéquate car elle ne s'adresse pas aux premiers produits résultant de la protéolyse. En effet, la plupart des travaux affirment aujourd'hui que la caséine $\beta$, substrat de la composante majeure du système enzymatique, donne par coupure de sa chaîne peptidique les caséines $\gamma_{1}$, $\gamma_{2}, \gamma_{3}$ et les protéoses-peptones $5,8 \mathrm{~S}$ et $8 \mathrm{~F}$. Ces produits ne sont pas tous solubles dans l'acide trichloracétique à $12 \%$.

Par ailleurs, dans une étude parallèle où nous cherchions à définir l'origine de l'activité protéinasique dans le lait, nous avons montré que cette protéolyse (libération du NPN) n'est due que pour 10 à $15 \%$ de sa valeur aux enzymes d'origine bactérienne. Là encore, il ne semble pas y avoir de corrélations évidentes (Humbert et al., 1982).

Les teneurs en germes des divers laits sont trop faibles pour induire une protéolyse conséquente due aux protéinases d'origine bactérienne. De plus, les différences de contamination entre ces laits sont trop minimes pour être pleinement significatives et nous aider à établir des corrélations. Dans l'expérience suivante, nous avons essayé de remédier à cet handicap.

\section{Lait cru contaminé}

La figure 3 rapporte la détermination de l'activité protéinasique dans un lait que nous avons appelé "stérile » (2500 germes/ml) et le même lait ayant subi une contamination bactérienne naturelle. L'analyse bactériologique a révélé qu'il contenait 60000 germes $/ \mathrm{ml}$ dont 14000 psychrotrophes.

L'activité enzymatique est plus élevée dans le lait contaminé que dans le lait témoin, pour 4 des 6 substrats étudiés. Elle est au moins égale au double pour L-Leu-4 na et L-Phe-4 na. Par contre, il n'y a pas de différence pour DL-Blpa et DL-Bapa.

Ces différences devraient nous servir à mettre au point une méthode d'estimation de l'origine de l'activité protéinasique fondée sur le calcul d'un ou plusieurs rapports d'activité entre 2 séries de substrats synthétiques répondant différemment. Reimerdes, Petersen et Kielwein (1979) ont effectué des essais dans cette optique en se limitant au calcul d'un rapport d'activité entre 2 substrats. Nous aimerions nous servir de rapports plus généraux concernant la microflore dans son ensemble et non une souche donnée. Ainsi, on pourrait peutêtre cerner la part due aux protéinases natives du lait et celle due aux enzymes d'origine bactérienne. Il ne faut toutefois pas perdre de vue le fait que le contenu en germes d'un échantillon de lait ne reflète pas la quantité et la spécificité des enzymes microbiens libérés dans le milieu. 


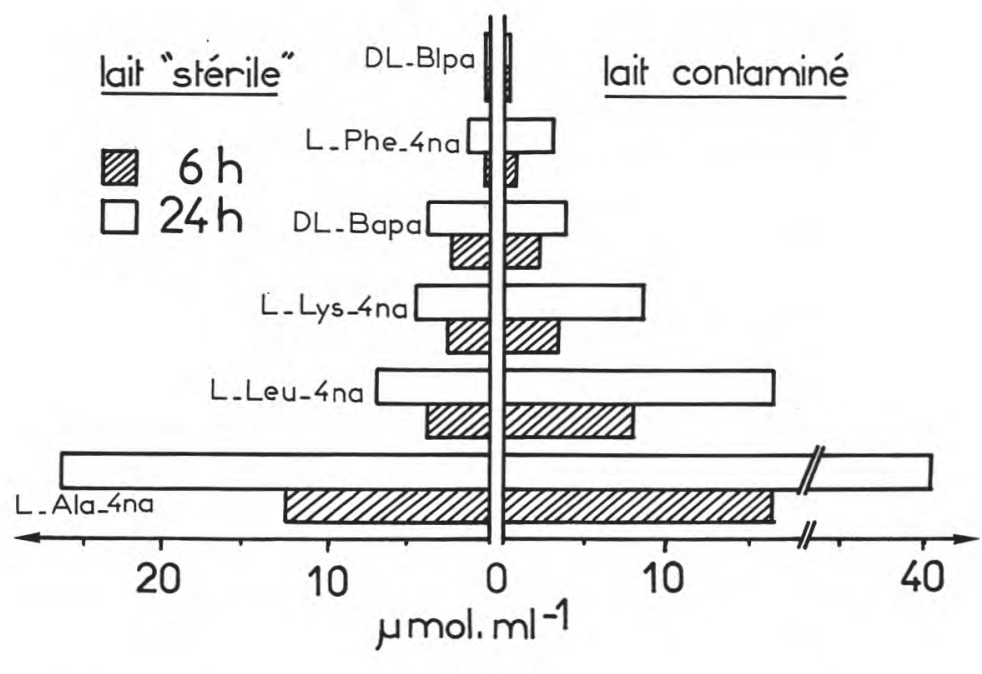

fig. 3

Activité protéinasique mesurée dans le lait cru "stérile » et dans le même lait après contamination microbienne naturelle (concentration en substrat : $5.10^{-3} \mathrm{~mol} / \mathrm{l}$ sauf pour L-Phe-4 na : $\left.3.10^{-3} \mathrm{~mol} / \mathrm{l}\right)$.

Proteinasic activity in "aseptic» and contamined raw milk (substrate concentration: $5.10^{-3} \mathrm{~mol} / \mathrm{l}$ except for L-Phe-4 na: $\left.3.10^{-3} \mathrm{~mol} / \mathrm{l}\right)$.

\section{CONCLUSION}

La méthode de dissolution des constituants colloïdaux du lait nous permet d'expérimenter directement dans le lait. Elle nous donne une approche complémentaire et intéressante de la détermination de l'activité protéinasique du lait avec différents substrats. Cette méthode permettra, sans traitement préalable, d'étudier la diversité de cette activité enzymatique et ceci dans de bonnes conditions de sensibilité et de précision.

\section{Remerciements}

Nous remercions $\mathrm{M}$. le Pr C. Alais pour ses précieux conseils dans la réalisation de ce travail. 


\section{Bibliographie}

Fox (P. F.) (1981). - Proteinases in dairy technology. Neth Milk Dairy J., 35, 233-253.

Humbert (G.) and Alais (C.) (1979). - Review of the progress of Dairy Science : The milk proteinase system. J. Dairy Res., 46, 559-571.

Humbert (G.), Brun-Bellut (J.), Mora (M. T.) et Alais (C.) (1982). - Soumis pour publication.

Kaminogawa (S.) et Yamauchi (K.) (1972). - Acid protease of bovine milk. Agric. Biol. Chem., 36, 2351-2356.

Linden (G.), Humbert (G.), Desnouveaux (R.) et Picard (J.) (1982). - Applications de la dissolution complète du lait à la détermination de quelques activités enzymatiques. Le Lait, 62, 209-219.

Noomen (A.) (1975). - Proteolytic activity of milk proteinase in raw and pasteurized cow's milk. Neth. Milk Dairy J., 29, 153-161.

Reimerdes (E. H.), Petersen (F.) und Kielwein (G.) (1979). - Milchproteinasen 9. Proteinasespektren von Caseinmicellen, Milchserum Rinderblutserum und Pseudomonas fluorescens. Milchwissenschaft, 34, 548-551.

Reimerdes (E. H.), Halpaap (I.) und Klostermeyer (H.) (1981). - Milchproteinasen 10. Enzymkinetischer Vergleich bei Rinderplasmin mit zwei Milchproteinasen. Milchwissenschaft, 36, 73-79.

Serres (L.), Amariglio (S.) et Petransxiène (D.) (1974). - Contrôle de la qualité des produits laitiers. Tome 2. Analyse microbiologique et analyse sensorielle. Ministère de l'Agriculture. Direction des Services Vétérinaires. Paris.

VISSER (S.) (1981). - Proteolytic enzymes and their action on milk proteins. A review. Neth. Milk. Dairy J., 35, 65-88. 